\title{
Semelhanças entre a doença idiopática do trato urinário inferior dos felinos e a cistite intersticial humana
}

\author{
Similarities between the feline idiopathic lower urinary tract disease \\ and human interstitial cystitis
}

\author{
Archivaldo Reche Junior ${ }^{1}$ Mitika Kuribayashi Hagiwara ${ }^{2}$
}

\section{- REVISÃO BIBLIOGRÁFICA -}

RESUMO

A doença idiopática do trato urinário inferior dos felinos (DITUIF) continua sendo um grande desafio para o clínico veterinário uma vez que, apesar dos avanços nas técnicas diagnósticas, sua etiologia continua por ser determinada. $O$ objetivo do presente trabalho é demonstrar as principais semelhanças entre a doença urinária felina e a cistite intersticial humana (CI), principalmente no que se refere à comprovação do caráter neurogênico da inflamação vesical, o papel do estresse na gênese elou exacerbação dos sinais clínicos da doença, e os principais achados cistoscópicos e histopatológicos em ambas as síndromes urinárias. Concluise, portanto, que além da dieta comercial, podem existir outros fatores com participação na inflamação vesical de felinos com DITUI.

Palavras-chave: gato, bexiga, doença urinária

\section{ABASTRACT}

Despite all the new diagnostic resources, the idiopathic feline lower urinary tract disease (iFLUTD) is still a problem to be solved in veterinary medicine. This review was carried out to compare the iFLUTD to interstitial cystitis, a painful bladder disease of human beings. The most important similarities of both diseases which will be discussed in the review are the urinary bladder neurogenic inflammation, the role of stress in the pathogenesis and/or exacerbation of clinical signs of both diseases (human and feline) and also the findings of the cystoscopy and histopathology. However, one can conclude that besides commercial diets, the might be some other explanation for the urinary bladder inflammation in cats with lower urinary tract disease.
Key words: cat, urinary bladder, urinary disease

\section{INTRODUÇÃO}

Os gatos domésticos são freqüentemente acometidos por doenças do trato urinário inferior que, embora possam ter causas diversas, caracterizam-se por resultarem em manifestações clínicas similares: hematúria, disúria, polaquiúria ou obstrução uretral. No entanto, esses sinais clínicos representam simplesmente uma resposta inespecífica de injúria das vias urinárias (OSBORNE et al., 1996).

Diferentemente do que se observa na espécie canina, na qual as doenças urinárias mais comuns são as infecções bacterianas associadas ou não aos cálculos de bexiga e/ou uretra, na grande maioria dos felinos não se consegue chegar ao agente causal, constituindo-se em verdadeiro desafio diagnóstico para o clínico. Portanto, a etiologia das doenças do trato urinário dos felinos pode ser multifatorial, complexa e, muitas vezes, indeterminada (OSBORNE et al., 1996; CHEW et al., 1997).

Durante muito tempo, Síndrome Urológica Felina (SUF) foi a denominação empregada para englobar as afecções das vias urinárias inferiores dos felinos, independentemente da causa. Atualmente, os termos SUF, cistite idiopática felina, DITUIF - Doença

${ }^{1}$ Professor Doutor do Departamento de Clínica Médica da Faculdade de Medicina Veterinária e Zootecnia (FMVZ), Universidade de São Paulo ( USP). Endereço para correspondência: Rua Agissê, 268 ap. 91, 05439-010, São Paulo. E-mail valdorec@uol.com.br

${ }^{2}$ Professor Titular (aposentado), Departamento de Clínica Médica, FMVZ - USP. 
Idiopática do Trato Urinário Inferior dos Felinos e mais recentemente cistite intersticial felina, são utilizados nos casos de doença do trato urinário em que se desconhece a causa da inflamação das vias urinária inferiores (ROSS, 1990; OSBORNE et al., 1999).

Nos últimos 30 anos, muito se tem estudado em relação ao envolvimento da dieta, o papel desempenhado pela presença de cristais e/ou cálculos e até mesmo de agentes virais no desenvolvimento da DITUIF. No entanto, poucos esforços têm sido direcionados para o estudo mais detalhado da inflamação da bexiga nesses pacientes.

Há pouco mais de setenta anos, surgiu o primeiro relato de gatos com doença urinária, em que se observava intensa cristalúria (KIRK, 1925), desde então inúmeros estudos têm tentado estabelecer qual o real significado da presença dos cristais, principalmente cristais de estruvita (fosfato amônio magnesiano), na etiopatogenia da DITUIF (TARTTELIN, 1987).

A adição de acidificantes urinários às dietas comerciais para gatos, nos últimos anos, propiciou uma diminuição relevante na precipitação de cristais e/ou crescimento de cálculos de estruvita sem, no entanto, diminuir a incidência da síndrome urinária. A explicação para tal fato pode estar relacionada a um aumento na casuística de cristalúria e/ou cálculos de oxalato de cálcio na população felina alimentada com dietas contendo acidificantes (OSBORNE et al., 1990; BUFFINGTON \& CHEW, 1999).

Atualmente, acredita-se que a cristalúria exerça um papel secundário na patogenia da DITUIF, ou seja, os cristais juntamente com restos celulares, originários da inflamação, seriam componentes imprescindíveis na formação dos tampões uretrais, os quais teriam vital importância na interrupção do fluxo urinário, principalmente nos machos, uma vez que estes apresentam uma uretra anatomicamente mais estreita quando comparada à das fêmeas (OSBORNE et al., 1992).

Além disso, são também freqüentes as recidivas da doença e tem-se observado que 35 a $50 \%$ dos gatos com DITUI, independentemente da terapia e da aparente recuperação, apresentam uma ou mais recidivas, principalmente nos seis primeiros meses após o episódio inicial (FERNÁNDEZ et al., 1989). Estudos prospectivos da doença urinária dos felinos puderam evidenciar a cronicidade do processo, associando às recidivas situações estressantes às quais o animal é submetido (CASTON, 1973; BUFFINGTON et al., 1996a; CLASPER, 1990).

De fato, a análise radiográfica das vias urinárias de gatos com quadro clínico de hematúria, disúria, polaquiúria ou obstrução uretral, pôde demonstrar espessamento marcante da parede vesical, o que poderia corroborar a teoria de que estes animais apresentam uma inflamação crônica da bexiga (RECHE \& HAGIWARA, 1998).

Nos últimos anos, têm-se observado uma possível semelhança entre a DITUIF e a Cistite Intersticial (CI) que acomete a espécie humana (BUFFINGTON et al., 1996a; CLASPER, 1990). Assim como na doença urinária felina, na grande maioria dos pacientes humanos, a etiologia da inflamação das vias urinárias é desconhecida (MESSING, 1987; RATNER, 2001). Em ambos os casos ocorre uma diminuição da excreção renal de glicosaminoglicana e alteração na permeabilidade epitelial da bexiga (BUFFINGTON et al., 1996b; GAO et al., 1994).

O diagnóstico da CI baseia-se, predominantemente, nos sinais clínicos que incluem disúria, polaquiúria, noctúria e intensa dor pré-púbica, normalmente não associados à infecção bacteriana das vias urinárias (MESSING, 1987). Os achados em cistoscopia também são importantes para o diagnóstico da CI, considerando-se que a observação de petéquias na submucosa de bexigas submetidas a hidrodistensão, durante o procedimento endoscópico, pode ser considerado um achado patognomônico da CI (MESSING \& STAMEY, 1978).

A patogenia da inflamação da bexiga, observada nos pacientes humanos com CI, tem sido amplamente estudada (MESSING \& STAMEY, 1978; HOLM-BENTZEN, 1989; JOHANSON \& FALL, 1994). Dentre as indagações concernentes à etiologia da CI, destacam-se os trabalhos que demonstram o caráter neurogênico da inflamação vesical (HAND, 1949; CHRISTMAS et al., 1990; HOHENFELLNER et al., 1992; PANG et al., 1995).

A evidenciação do aumento na densidade de fibras nervosas sensoriais contendo o neurotransmissor-neuropeptídeo Substância P (SP), assim como de fibras nervosas simpáticas, na lâmina própria e camada muscular, da bexiga de pacientes com CI, vem corroborar a hipótese de se tratar de inflamação neurogênica (HOHENFELLNER et al., 1992; PANG et al., 1995). Estudos recentes puderam comprovar que a bexiga dos gatos com DITUIF também apresenta um maior número de fibras sensoriais contendo o neuropeptídeo Substância P (SP) (CAITO, 1995), à semelhança do que se observa nos pacientes humanos.

O sistema nervoso simpático (SNS) parece exercer um papel importante na fisiopatogenia tanto da DITUIF como da CI humana. Sabe-se que o SNS, em condições normais, não participaria da excitação 
de fibras nervosas sensoriais. No entanto, a observação do aparecimento de sinais clínicos da doença urinária frente a situações estressantes, tanto nos pacientes humanos como nos gatos, sugere uma possível interação entre SNS e neurônios sensoriais localizados na bexiga (HOHENFELLNER et al., 1992; BUFFINGTON \& PACAK, 2001).

A etiologia do processo inflamatório vesical, observado tanto nos gatos como em pessoas, é, até o presente momento, desconhecida. Enquanto se avança no conhecimento da patogenia da CI, não se observa a mesma evolução nos estudos da DITUIF, a despeito da dedicação de inúmeros pesquisadores.

O objetivo do presente trabalho é detalhar as comprovadas semelhanças entre a DITUIF e a CI em pessoas.

\section{DESENVOLVIMENTO}

\section{O PAPEL DO ESTRESSE NA ETIOPATOGENIA DA DITUIF E DA CI}

A evidenciação de que tanto os felinos com DITUI quanto pessoas com CI apresentam um aumento no número de fibras simpáticas na bexiga, além de contribuir para a teoria de que o processo inflamatório vesical teria um caráter neurogênico (HOHENFELLNER et al., 1992; RECHE, 1998), poderia, também, definir uma provável participação do sistema nervoso autonômico-simpático na patogenia de ambas as doenças urinárias.

Outro aspecto bastante interessante e que, de certa forma, demonstra o envolvimento do sistema nervoso simpático (SNS) na patogenia das doenças urinárias felina e humana é a observação da recrudescência ou agravamento dos sinais frente a situações de estresse (CASTON, 1973; WHITMORE, 1994; LUTGENDORF et al., 2001; ROTHROCK et al., 2001a; ROTHROCK et al., 2001b). Mudanças bruscas no manejo ambiental, viagens, introdução de novos animais ou mesmo moradores na propriedade, participações em exposições e até mesmo mudanças climáticas podem ser consideradas situações estressantes para alguns gatos (CASTON, 1973).

Sabe-se que as atividades periféricas do SNS são controladas centralmente pelo complexo locus coeruleus (LC) e pelo núcleo paraventricular (PVN), localizados, respectivamente, na ponte e no hipotálamo. A noradrenalina (NA) é o principal neurotransmissor, produzido e liberado pelas células que compõem o LC, e o hormônio liberador de corticotrofina $(\mathrm{CRH})$ o principal componente do núcleo PVN. A freqüente co-ativação do SNS e do complexo LC é, em condições normais, funcionalmente significante, e pode refletir o fato de que o núcleo paragigantocelular (PGi), localizado na medula oblonga, inerva tanto o complexo LC como os núcleos pré-ganglionares simpáticos, localizados no corno lateral da medula espinal (ELAM et al., 1986).

Tem-se demonstrado que o estresse agudo e o crônico aumentam a imunorreatividade da tirosina hidroxilase (IR-TH), enzima limitante da taxa da síntese de catecolaminas, nos neurônios adrenérgicos do complexo LC de animais saudáveis (ZIGMOND et al., 1995). Pouco se sabe sobre os efeitos do estresse induzido por enfermidades crônicas, de ocorrência natural, nos neurônios NA-LC, como é o caso da DITUIF e da CI.

A avaliação imunohistoquímica do complexo locus coeruleus (LC) de gatos com DITUI, na fase quiescente ou assintomática da doença demonstrou um aumento bastante significante da imunorreatividade para a enzima tirosina hidroxilase (TH-IR). Tal achado, segundo os autores do referido estudo, poderia sugerir a existência de uma população de felinos que apresentam uma hiperatividade do SNS e que, aliada a outros fatores predisponentes, poderia determinar o aparecimento, ou mesmo o agravamento dos sinais clínicos da doença urinária felina (RECHE \& BUFFINGTON, 1998).

Curiosamente, CHEW et al. (1997) observaram que o antidepressivo tricíclico amitriptilina, quando utilizado em felinos com sinais clínicos de DITUI, apresentaram remissão parcial ou total desses sinais. De fato, os fármacos antidepressivos, através de diferentes mecanismos de ação, acabam por determinar uma diminuição na atividade das células do complexo LC, levando a uma diminuição dos níveis de noradrenalina circulante no SNC. Portanto, concluise que as células noradrenérgicas do complexo LC devem ter participação importante na perpetuação ou mesmo exacerbação dos sinais clínicos observados em gatos com DITUI.

Uma vez comprovada a piora de ambas as doenças urinárias frente a situações estressantes às quais os pacientes são submetidos, deve-se recomendar a todos os proprietários de gatos com DITUI que evitem submeter seus animais a condições potencialmente adversas, o que pode ser bastante complicado uma vez que uma simples mudança na dieta ou manejo higiênico podem ser estressantes para alguns gatos.

\section{INFLAMAÇÃO NEUROGÊNICA DA BEXIGA EM GATOS COM DITUI E PESSOAS COM CI}

Dentre as teorias propostas para explicar a inflamação vesical na DITUIF e na CI, incluem-se as 
infecções virais, doença mediada por mastócitos e defeito na camada superficial da mucosa urinária de glicosaminoglicana (BUFFINGTON et al., 1996b; PARSONS, 1986). Merecem destaque as evidências de que o processo inflamatório vesical tanto de gatos com DITUI como de pessoas com CI, seja mediado por neurotransmissores liberados de fibras nervosas vesicais aferentes e/ou eferentes determinando um caráter neurogênico à inflamação vesical, em ambas as espécies (HAND, 1949; HOHENFELLNER et al., 1992; PANG et al., 1995; CAITO, 1995; WOLFE et al., 1995).

Os primeiros relatos da participação do sistema nervoso na patogenia da CI datam de 1949, quando foram estudados 223 pacientes com a doença urinária, observando-se um aumento no número de fibras nervosas na lâmina própria da bexiga dos mesmos (HAND, 1949). Em estudo realizado em 1957, cogita-se que a dor suprapúbica descrita pelos pacientes com CI, seria decorrente de uma possível doença nas raízes nervosas sacrais e que a rizotomia sacral poderia beneficiar esses pacientes (BOHM \& FRANKSSON, 1957). Ainda, a denervação extra-vesical através da cistólise passa a ser considerada uma alternativa terapêutica para diminuir a dor suprapúbica dos pacientes com CI (WORTH \& TURNERWARWICK, 1973).

Ainda se observa que pacientes com CI apresentam um aumento no número de fibras nervosas na bexiga, mais precisamente na região subepitelial e camada muscular, o que normalmente não é observado em outras formas de cistite crônica (CHRISTMAS et al., 1990).

A provável participação dos neurônios sensoriais e do neuropeptídeo substância P (SP), na etiopatogenia da CI, tornou-se mais evidente a partir de 1995, quando da constatação de que pacientes com CI apresentam um grande número de fibras nervosas sensoriais, contendo o neuropeptídeo SP, na lâmina própria da bexiga, e que estas dispõem-se muito próximas dos mastócitos. Tal fato permite vislumbrar uma provável interrelação entre SP, mastócitos e a patogenia da CI (PANG et al., 1995). Estudos realizados na bexiga de gatos com DITUI evidenciaram também um aumento na densidade de fibras nervosas sensoriais, contendo o neuropeptídeo SP, assim como um significante aumento na concentração dos receptores para SP, NK-2 (CAITO, 1995; WOLFE et al., 1995).

Pesquisas concernentes à inflamação neurogênica demonstram que as fibras nervosas sensoriais parecem interagir de alguma forma com as fibras eferentes simpáticas (LEVINE et al., 1986), o que não ocorre em condições normais. De fato, maior número de fibras eferentes catecolaminérgicas na bexiga, têm sido demonstradas, tanto em pessoas com CI, como em gatos com DITUI (HOHENFELLNER et al., 1992; RECHE, 1998; PEEKER et al., 2000).

No entanto, nenhum estudo foi capaz de definir, efetivamente qual a real participação das fibras nervosas sensoriais e eferentes que se encontram em maior número tanto na bexiga de gatos com DITUI como de pessoas com CI. Essa neuroplasticidade vesical seria uma causa ou consequência da inflamação? Portanto, antes de se classificar definitivamente a inflamação como neurogênica, deverse-ia esclarecer a real participação dessas fibras nervosas e seus neurotransmissores em ambas as doenças urinárias.

\section{EXAME CISTOSCÓPICO DE GATOS COM DITUI E PESSOAS COM CI}

No exame cistoscópico da bexiga de felinos que apresentavam sinais clínicos da DITUI, foram observados edema de mucosa e presença de petéquias na lâmina própria, sugerindo-se dessa forma o comprometimento vesical (BUFFINGTON \& CHEW, 1993).

Pacientes humanos com CI, submetidos a cistoscopia, também apresentam edema de mucosa e petéquias em lâmina própria, à similitude do que se observa nos gatos com DITUI. No entanto, embora alguns autores considerem essas alterações patognomônicas da CI (MESSING \& STAMEY, 1978), outros consideram-nas achados inespecíficos, podendo estar presentes em qualquer outra doença urinária como cistites bacterianas e tumores em bexiga (JOHANSSON \& FALL, 1990).

Portanto, muito embora a cistoscopia seja um método diagnóstico pouco invasivo, as informações obtidas são inespecíficas e, conseqüentemente, de pouca valia no diagnóstico de ambas as doenças urinárias. Talvez, a maior importância da cistoscopia, tanto na medicina veterinária como na humana, seja a facilidade na obtenção de material para análise histológica, contribuindo provavelmente com informações importantes na classificação da inflamação vesical desses pacientes.

\section{HISTOPATOLOGIA DA BEXIGA DE GATOS COM DITUI E PESSOAS COM CI}

Dentre os achados histopatológicos mais freqüentemente descritos para os humanos com CI destacam-se: a inflamação perineural e perivascular, aumento na densidade de mastócitos tanto na lâmina 
própria como na camada muscular, edema da lâmina própria, hiperplasia epitelial, ulcerações epiteliais, maior número de vasos sangüíneos na lâmina própria e fibrose (JOHANSSON \& FALL, 1990).

Curiosamente, são raras as publicações disponíveis sobre a histopatologia da DITUIF. Nos poucos trabalhos em que a análise histopatológica da bexiga de gatos com DITUI foi realizada, observou-se um quadro histológico bastante semelhante ao da CI, ou seja, pronunciado edema da lâmina própria, infiltrado inflamatório mononuclear, aumento no número de mastócitos na mucosa vesical, pregueamento epitelial, petéquias subepiteliais e inflamação perineural (BUFFINGTON \& CHEW, 1993; OSBALDISTON \& TAUSSIG, 1970; RECHE \& HAGIWARA, 2001).

A infiltração de mastócitos na bexiga de ambas as espécies, permite tecer algumas considerações quanto ao mecanismo etiopatogênico da inflamação vesical. Na CI, os mastócitos respondem por cerca de $20-65 \%$ do infiltrado celular, na dependência da existência ou não de úlceras (ENERBACK et al., 1989). Em cortes histológicos da bexiga de felinos com DITUI, também já foi descrito um maior número de mastócitos (BUFFINGTON \& CHEW, 1993). Outra importante evidência da presença de mastócitos na bexiga de pessoas com CI ou de gatos com DITUI, está na demonstração de um aumento na liberação de histamina após a hidrodistensão da bexiga (BUFFINGTON \& CHEW, 1993; THEOHARIDES et al., 1995). A participação dos mastócitos na etiopatogenia da CI e, mais recentemente, da DITUIF, vem recebendo especial atenção nos últimos anos, pois se sabe que as substâncias liberadas por essas células podem estar envolvidas no desenvolvimento de muitas das alterações histológicas e dos sinais clínicos relacionados a ambas as doenças (BUFFINGTON \& CHEW, 1993; SANT, 1997). A base fisiológica para explicar a ocorrência das petéquias ou hemorragias subepiteliais na bexiga de gatos com DITUI e humanos com CI poderia ser atribuída também à ativação de mastócitos, e conseqüente liberação de substâncias vasoativas como histamina, prostaglandinas e leucotrienos (RECHE, 1998).

A escassez de publicações sobre o assunto, em medicina veterinária, em parte pode ser explicada pelas dificuldades na realização de biópsias das vias urinárias na clínica de pequenos animais. No entanto, a recente introdução de técnicas diagnósticas mais sofisticadas, como a cistoscopia, possibilitará ao clínico veterinário obter amostras da bexiga desses pacientes e, conseqüentemente, facilitará a realização de estudos prospectivos da doença, assim como estudos de correlação clinico-patológica.

\section{PAPEL DAS GLICOSAMINOGLICANAS (GAGs) NA ETIOPATOGENIA DA DITUIF E DACI}

Os gatos com DITUI parecem apresentar uma diminuição significante da excreção urinária das glicosaminoglicanas (GAGs), substâncias que podem exercer importante função de proteção ao epitélio vesical, controlando sua permeabilidade (BUFFINGTON et al., 1996b). A diminuição qualitativa e quantitativa da camada das glicosaminoglicanas, também é considerada um achado freqüente entre os pacientes humanos com CI. Portanto, o baixo nível urinário das GAGs, normalmente, está associado a um aumento na permeabilidade desse epitélio, permitindo que constituintes da urina cheguem às camadas subepiteliais e, conseqüentemente, induzam o aparecimento do processo inflamatório (PARSONS, 1986). A permeabilidade epitelial da bexiga de gatos com DITUI foi recentemente avaliada através da administração intravesical de salicilato de sódio, com posterior mensuração dos níveis do fármaco na corrente sangüínea. Observou-se que a concentração plasmática do salicilato foi sete a oito vezes maior do que a observada em gatos normais (GAO et al., 1994).

Mais uma vez, a simples identificação da diminuição das GAGs na bexiga de pessoas com CI e gatos com DITUI parece não ser suficiente para elucidar as dúvidas concernentes à inflamação vesical em ambas as espécies. Informações importantes ainda precisam ser obtidas para um melhor esclarecimento da participação das GAGs em ambas as doenças urinárias, tais como os tipos mais frequentes das GAGs em felinos e humanos, se ocorre diminuição seletiva de alguma(s) GAGs e principalmente, se ocorre alterações quali e quantitativas das GAGs em outras doenças urinárias inferiores tanto em gatos como em pessoas.

\section{CONCLUSÕES}

Considerando-se os resultados dos últimos e mais recentes estudos concernentes à DITUIF, contempla-se uma nova e promissora era para a melhor compreensão do mecanismo etiopatogênico envolvendo a doença urinária felina, abrindo-se novos caminhos para as pesquisas que, nos últimos anos, voltaram-se quase que exclusivamente ao estudo do papel das dietas comerciais no aparecimento dessa afecção. 


\section{REFERÊNCIAS BIBLIOGRÁFICAS}

BOHM, E.; FRANKSSON, C. Interstitial cystitis and sacral rhizotomy. Acta Chirurgica Scandinavica, v.113, n.2, p. 63-67, 1957.

BUFFINGTON, C.A.T.; CHEW, D.J. Presence of mast cells in submucosa and detrusor of cats with idiopathic lower urinary tract disease. Journal of Veterinary Internal Medicine, v.7, n.2, p. 126,1993

BUFFINGTON, C.A.T.; CHEW, D.J.; DIBARTOLA, S.P. Interstitial cystitis in cats. Veterinary Clinics of North America: Small Animal Practice, v.26, n.2, p.317-326, 1996a.

BUFFINGTON, C.A.T. et al. Decreased urine glycosaminoglycan excretion in cats with interstitial cystitis. The Journal of Urology, v.155, n.5, p.1801-1804, 1996b.

BUFFInGTON, C.A.T.; CHEW, D.J. Calcium oxalate urolithiasis in cats. Journal of Endourology, v.13, n.9, p.659-663, 1999.

BUFFINGTON, C.A.T.; PACAK, K. Increased plasma norepinephrine concentration in cats with interstitial cystitis. The Journal of Urology, v.165, n.6Pt1, p.2051-2054, 2001.

CAITO, M. Neuron-specific enolase and substance $P$ immunoreactivity in urinary bladder of cats with idiopathic cystitis. 1995. 63f. Dissertation (Master in Clinical Sciences) - College of Veterinary Medicine, The Ohio State University.

CASTON, H.T. Stress and the feline urological syndrome. Feline Practice, v.4, p.14-22, 1973.

CHEW, D.J. et al. Management of nonobstructive lower urinary tract disease in cats. A Supplement to Compendium on Continuing Education for the Practicing Veterinarian, v.19, n.3, p.53-61, 1997.

CHRISTMAS, T.J. et al. Nerve fibre proliferation in interstitial cystitis. Virchows Archives A Pathological Anatomy and Histopathology, v.416, n.5, p.447-451, 1990.

CLASPER, M. A case of interstitial cystitis and Hunner's ulcer in a domestic shorthaired cat. New Zealand Veterinary Journal, v.38, n.4, p.158-160, 1990.

ELAM, M.; THORÉN, P.; SVENSSON, T.H. Locus coeruleus neurons and sympathetic nerves: activation by visceral afferents. Brain Research, v.375, n.1, p.117-125, 1986.

ENERBACK, L.; FALL, M.; ALDENBORG, F. Histamine and mucosal mast cells in intertitial cystitis. Agents and Actions, v.27, n.1-2, p.113-116, 1989.

FERNÁNDEZ, F.; LUERA, M.; PRATS, A. Situación actual del complejo urinario del gato. In: FERNÁNDEZ, F. et al. Actualidad del complejo urinario del gato. Barcelona : Fundacion Purina, 1989. p.59-96.

GAO, X.; BUfFington, C.A.T.; AU, J.L. Effect of interstitial cystitis on drugs absorption from urinary bladder. The Journal of Pharmacology and Experimental Therapeutics, v.271, n.2, p.818-823, 1994.
HAND, J.R. Interstitial cystitis: report of 223 cases (204 women and 19 men). The Journal of Urology, v.61, p. 291310,1949 .

HOHENFELLNER, M. et al. Interstitial cystitis: increased sympathetic innervation and related neuropeptide synthesis. The Journal of Urology, v.147, n.3, p.587-591, 1992.

HOLM-BENTZEN, M. Pathology and pathophysiology of painful bladder diseases. Urologia Internationalis, v.44, n.6, p.327-331, 1989.

JOHANSSON, S.L.; FALL, M. Clinical features and spectrum of light microscopic changes in interstital cystitis. The Journal of Urology, v.143, n.6, p.1118-1124, 1990.

JOHANSSON, S.L.; FALL, M. Pathology of interstitial cystitis. Urologic Clinics of North America, v.21, n.1, p.55-62, 1994.

KIRK, H. The diseases of the cat. In: genital diseases. Chicago : Eger, 1925. p.261-273.

LEVINE, J.D. et al. Contribution of sensory afferents and sympathetic efferents to joint injury in experimental arthritis. The Journal of Neuroscience, v.6, n.12, p.3423-3429, 1986.

LUTGENDORF, S.K. et al. Laboratory stress model for examining stress and symptomatology in interstitial cystitis patients. Urology, v.57, n.6 Suppl 1, p.122, 2001.

MESSING, E.M.; STAMEY, T.A. Interstitial cystitis: early diagnosis, pathology and treatment. Urology, v.12, n.4, p.381392, 1978 .

MESSING, E.M. The diagnosis of intertitial cystitis. Urology, v.29, n.4S, p.4-7, 1987.

OSBALDISTON, G.W.; TAUSSIG, R.A. Clinical report on 46 cases of feline urological syndrome. Veterinary Medicine \& Small Animal Clinician, v.65, n.5, p.461-468, 1970.

OSBORNE, C.A. et al. Medical dissolution of feline struvite urocystoliths. Journal of the American Veterianry Medical Association, v.196, n.7, p.1053-1060, 1990.

OSBORNE, C.A.; KRUGER, J.M.; LULICH, J.P. Feline matrixcrystalline urethral plugs: a unifying hypothesis of causes. Journal of Small Animal Practice, v.33, n.4 , p.172-177, 1992.

OSBORNE, C.A.; KRUGER, J.M.; LULICH, J.P. Feline lower urinary tract disorders: definition of terms and concepts. Veterinary Clinics of North America: Small Animal Practice, v.26, n.2, p.169-179, 1996.

OSBORNE, C.A. et al. Feline urologic syndrome, feline lower urinary tract disease, feline interstitial cystitis: What's in a name? Journal of the American Veterinary Medical Association, v.214, n.10, p.1470-1480, 1999.

PANG, $X$. et al. Increased number of substance $P$ positive nerve fibres in interstitial cystitis. British Journal of Urology, v.75, n.6, p.744-750, 1995.

PARSONS, C.L. Bladder surface glycosaminoglycan: efficient mechanism of environmental adaptation. Urology, v.27, n.S2, p.9-14, 1986. 
PEEKER, R. et al. Increased tyrosine hydroxylase immunoreactivity in bladder tissue from patients with classic and nonulcer interstitial cystitis. The Journal of Urology, v. 163, n.4, p.1112-1115, 2000.

RATNER, V. Interstitial cystitis: a chronic inflammatory bladder condition. World Journal of Urology, v.19, n.3, p.157-159, 2001 .

RECHE JR, A. Contribuição do sistema nervoso simpático na etiopatogenia da síndrome urológica felina (SUF) 1998. 115f. Tese (Doutorado em Clínica Veterinária) - Faculdade de Medicina Veterinária e Zootecnia, Universidade de São Paulo.

RECHE JR, A.; BUFFINGTON, C.A.T. Increased tyrosine hydroxylase immunoreactivity in the locus coeruleus of cats with interstitial cystitis. The Journal of Urology, v.159, p.1045-1047, 1998.

RECHE JR, A.; HAGIWARA, M.K. Estudo clínico da doença do trato urinário inferior dos felinos em gatos domésticos de São Paulo. Brazilian Journal of Veterinary Research and Animal Science, v.35, n.2, p.69-74, 1998.

RECHE JR, A.; HAGIWARA, M.K. Histopatologia e morfometria da bexiga de gatos com doença idiopática do trato urinário inferior (DITUI). Ciência Rural, v.31, n.6, p.10451049, 2001

ROSS, L.A. Feline urologic syndrome: understanding and diagnosing this enigmatic disease. Veterinary Medicine, v.85, n.11, p.1194-1222, 1990.

ROTHROCK, N.E. et al. Daily stress and symptom exacerbation in interstitial cystitis patients. Urology, v.57, n.6 Suppl.1, p.122, 2001a.
ROTHROCK, N.E. et al. Stress and symptoms in patients with interstitial cystitis: a life stress model. Urology, v.57, n.3, p.422-427, $2001 \mathrm{~b}$.

SANT, G.R. Interstitial cystitis. Current Opinion in Obstetrics and Gynecology, v.9, n.5, p.332-336, 1997.

TARTTELIN, M.F. Feline struvite urolithiasis: factors affecting urine $\mathrm{pH}$ may be more important than magnesium levels in food. Veterinary Record, v.121, n.11, p.245-248, 1987.

THEOHARIDES, T.C. et al. Activation of bladder mast cells in intersttital cystitis: a light and electron microscopic study. The Journal of Urology, v.153, n.3, Pt 1, p.629-636, 1995.

WHITMORE, K.E. Self-care regimens for patients with interstitial cystitis. Urologic Clinics of North America, v.21, n.1, p.121-130, 1994

WOLFE, S.A.; SOKOLOV, A.G.; BUFFINGTON, C.A T. Effects of interstitial cystitis on bladder and spinal cord tachykinin receptor concentrations in cats. In: MEETING OF THE SOCIETY FOR NEUROSCIENCE, 1995, San Diego, CA. Proceedings... San Diego : Society for Neuroscience, 1995. p. 123 .

WORTH, P.H.; TURNER-WARWICK, R. The treatment of interstitial cystitis by cystolisis with observations on cystoplasty. British journal of Urology, v.45, n.1, p.65-71, 1973.

ZIGMOND, M.J.; FINLAY, J.M.; SVED, A.F. Neurochemical studies of central noradrenergic responses to acute and chronic stress-implications for normal and abnormal behavior. In: FRIDMAN, M.J.; CHARNEY, D.S.; DEUTCH, A.Y. Neurobiological and clinical consequences of stress from normal adaptation to post-traumatic stress disorder. Philadelphia : Lippincott-Raven, 1995. p.45-60. 